\title{
Qualitative behaviour of some simple networks
}

\author{
$\mathrm{J}$ Feng $\nmid$ and K P Hadeler $\ddagger$ \\ $\dagger$ Statistics Group, The Babraham Institute, Babraham, Cambridge CB2 4AT, UK, and \\ Mathematisches Institut, Universität München, München, Germany \\ $\ddagger$ Biomathematik, Universität Tübingen, Tübingen, Germany
}

Received 14 November 1995

\begin{abstract}
Some classical neural network systems including the Hartline-Ratliff system, the Linsker system, and the general sigmoid dynamics, are reconsidered within a more general class of dynamical systems. For synchronous dynamics the existence, uniqueness, local and global stability of stationary points is investigated. For asynchronous dynamics a convergence theorem is proved. The application of the theory of quasimonotone flows leads to some insights so far not widespread in network theory.
\end{abstract}

\section{Introduction}

Since the first neural networks appeared fifty years ago (McCulloch-Pitts [25]) as nets of $(0,1)$-neurons there has been a tendency to provide these systems with more structure: continuous state, continuous time, delays, stochastic actions, etc. By now it seems difficult to separate network theory from dynamical systems, or stochastic processes. There are differences in notation, though. Whereas in dynamical systems one tries to present results in coordinate-free form, in a general qualitative setting, network theory uses coordinates representing (the states of) single units or 'neurons'. Where dynamical systems describe nonlinearities by their qualitative features (monotone, concave, etc), networks use concrete examples. The most important difference results from the degree of smoothness required. In dynamical systems one usually studies smooth mappings and vector fields and only occasionally does one introduce a discrete caricature as a model example. In network theory there are many piecewise linear and even piecewise constant functions that lead to a-false-impression that the systems are more explicit, easier to analyse, or easier to simulate. However, the tools of calculus are powerful, and any degree of discreteness is an obstacle to a thorough analysis.

In this review we try to give a unified analysis to some networks that have appeared in the literature, and we try to connect this approach to the present theory of dynamical systems. The results apply to the Hartline-Ratliff system [10, 13, 31], the Linsker dynamics $[6-8,22]$, and a general sigmoid dynamics $[1,14]$. These networks are characterized by three properties: there is a linear operation given by a matrix, there is a 'simple' nonlinear function which makes the network a non-trivial dynamical system, and there is monotonicity with respect to a partial ordering of the underlying space. This latter aspect is the most important property. Together with the possibility of asynchronous dynamics it is the only justification to use a coordinate notation, and it is probably the only way to establish a connection to non-trivial results from dynamical systems theory. The networks will be dynamical systems in $\mathbb{R}^{n}$, with the usual coordinate notation. 


\section{The systems}

Here we define the system to be studied. There is a family of scalar functions $f_{j}: \mathbb{R} \rightarrow \mathbb{R}$, $j=1, \ldots, n$ that describe the local action of the neurons. These functions are assumed Lipschitz continuous and non-decreasing.

There is a real vector $b \in \mathbb{R}^{n}, b=\left(b_{j}\right)$. Its components can be considered as 'inputs'. Finally there is a real matrix $A=\left(a_{j k}\right)$ of order $n$ that describes the interaction between the neurons.

Discrete time is denoted $t=0,1,2, \ldots$, continuous time $t \in \mathbb{R}$. The discrete time variable will be denoted as a superscript $x^{t}$ or as an argument $x(t)$, the continuous time variable always as $x=x(t)$.

We start with coordinate notation and then pass to a more convenient semi-abstract setting. In coordinate notation the discrete time system is

$$
x_{j}^{t+1}=\sum_{k=1}^{n} a_{j k} f_{k}\left(x_{k}^{t}\right)+b_{j} \quad j=1, \ldots, n \quad t=0,1,2, \ldots
$$

We introduce the 'Nemytskij operator' $f: \mathbb{R}^{n} \rightarrow \mathbb{R}^{n}$ acting coordinate-wise

$$
(f(x))_{j}=f_{j}\left(x_{j}\right) \quad j=1, \ldots, n .
$$

Then the system (1) reads

$$
x^{t+1}=A f\left(x^{t}\right)+b .
$$

If $f$ has identical components then we use the same symbol for the vector and the scalar function, $f=(f, \ldots, f)$. In the corresponding continuous time system we introduce a time constant $\tau>0$,

$$
\tau \dot{x}+x=A f(x)+b .
$$

In the older literature much attention has been paid to the fact that (3) can be written in equivalent form

$$
y^{t+1}=f\left(A y^{t}+b\right) .
$$

The correspondence is established by the transformation

$$
\begin{aligned}
& y=f(x) \\
& x=A y+b .
\end{aligned}
$$

This equivalence is somewhat unexpected, since neither (6) nor (7) need to be invertible. It may be worth the effort to study this phenomenon in greater detail.

Consider two functions $F, G: \mathbb{R}^{n} \rightarrow \mathbb{R}^{n}$ and two discrete dynamical systems

$$
\begin{aligned}
x^{t+1} & =G\left(F\left(x^{t}\right)\right) & t & =0,1,2, \ldots \\
y^{t+1} & =F\left(G\left(y^{t}\right)\right) & t & =0,1,2, \ldots .
\end{aligned}
$$

If $\left\{x^{t}\right\}$ is a trajectory of (8), then $\left\{y_{i}^{t}\right\}, y^{t}=F\left(x^{t}\right)$, is a trajectory of (9). If $\left\{y^{t}\right\}$ is a trajectory of (9) then $\left\{x^{t}\right\}, x^{t}=G\left(y^{t}\right)$ is a trajectory of (8). 
On the other hand, from $x^{0}$ one gets $y^{0}=F\left(x^{0}\right)$, then $G\left(y^{0}\right)=G\left(F\left(x^{0}\right)\right)$ which is $x^{1}$ (and not $x^{0}$ ). Thus the systems (8) and (9) are not conjugate. Going from $x$ to $y$ and then back to $x$ leads to the dynamical system (8) on the image set of $G \circ F$. Nevertheless, equations (8), (9) have essentially (after the first step) the same trajectories, limit sets, etc.

If the functions $f_{j}$ are invertible and differentiable then a similar transition is possible in the system (4), leading to

$$
\tau \dot{y}=f^{\prime}\left(f^{-1}(y)\right)\left(A y-b-f^{-1}(y)\right) .
$$

Here $f^{\prime}$ is the derivative of $f$, and $f^{-1}$ is the inverse function.

In view of the coordinate notation it makes sense to introduce coordinate norms in the form of weighted sum norms

$$
\|x\|_{\alpha}=\sum_{j=1}^{n} \alpha_{j}\left|x_{j}\right|
$$

where $\alpha=\left(\alpha_{1}, \ldots, \alpha_{n}\right)$ is a vector of positive components.

If nothing else is said, then $\alpha=(1, \ldots, 1)$,

$$
\|x\|=\sum_{j=1}^{n}\left|x_{j}\right| .
$$

We assume that the functions $f_{j}$ have a global Lipschitz bound $L_{j}$,

$$
\left|f_{j}(u)-f_{j}(v)\right| \leqslant L_{j}|u-v| .
$$

Let

$$
L=\max _{1 \leqslant j \leqslant n} L_{j} .
$$

Then the 'right-hand side' of the differential equation (4), i.e.

$$
\dot{x}=\frac{1}{\tau}[A f(x)+b-x]
$$

has a global Lipschitz bound. Therefore the solutions of the system (4) exist for all time.

We shall consider the special cases where the matrix $A$ has non-negative or non-positive entries. Then we use the usual partial ordering of the space $\mathbb{R}^{n}$ induced by the cone $\mathbb{R}_{+}^{n}=\left\{x \in \mathbb{R}^{n}: x \geqslant 0\right\}$. Thus $x \geqslant y$ if $x_{j} \geqslant y_{j}$ for $j=1, \ldots, n$. If the matrix $A$ has non-positive entries, instead of equation (5) we write

$$
y^{t+1}=f\left(c-B y^{t}\right)
$$

with a non-negative matrix $B=\left(b_{j k}\right), b_{j k} \geqslant 0$, and $c=\left(c_{j}\right)$. The corresponding continuous time system is

$$
\tau \dot{x}+x=-B f(x)+c .
$$

An important quantity is the spectral radius of a matrix, i.e. the maximal modulus of any eigenvalue of that matrix. The spectral radius of a matrix $B$ is called $\rho(B)$.

For any matrix $B=\left(b_{j k}\right)$ we introduce the absolute value of that matrix, $|B|=\left(\left|b_{j k}\right|\right)$. It is known that $\rho(B) \leqslant \rho(|B|)$ (see [2]).

Now we proceed to the qualitative analysis of the systems $(3)=(5)$ and (4). 


\section{Dissipativeness and stationary points}

The system is called dissipative if there is a compact invariant set $M$ such that for every $y^{0} \in \mathbb{R}^{n}$ there is a finite $t$ such that $y^{t} \in M$.

Proposition 1. Suppose one of the following hypotheses is satisfied.

(i) The functions $f_{i}$ are uniformly bounded.

(ii) The matrix $A$ has non-negative entries and the functions $f_{i}$ have uniform upper bounds. (iii) The matrix $A$ has non-positive entries and the functions $f_{j}$ have uniform lower bounds. Then the system (5) is dissipative.

Proof. The first assertion is trivial. The second and third are similar. We show the third for the system (16). Let $f_{j}(x) \geqslant a_{j}$ for $j=1, \ldots, n$ and all $x$. Let $a=\left(a_{j}\right)$. For $y \in \mathbb{R}^{n}$, we have $f(c-B y) \geqslant a$, and $f(c-B a-B(f(c-B y)-a)) \leqslant f(c-B a)$. Thus $\mathbb{R}^{n}$ is mapped into the compact convex set $M=\{x: a \leqslant x \leqslant f(c-B a)\}$.

Stationary solutions $x$ of the systems (3) and (4) satisfy the equation

$$
x=A f(x)+b .
$$

Corollary 2. If any of the assumptions of proposition 1 is satisfied then the corresponding system (5) or (16), (4) or (17), has a stationary point.

Proof. Apply Brouwer's fixed point theorem to the set $M$.

Proposition 3. Let $\rho=\rho(|A|)$ be the spectral radius of the matrix $|A|$ and let $L$ be the global Lipschitz constant for $f$. Assume $\rho L<1$. Then the following is true.

(i) For any vector $b$, equation (18) has a unique solution $\bar{x}_{b}$.

(ii) $\bar{x}_{b}$ is a global attractor for the system (3).

(iii) $\bar{x}_{b}$ is a global attractor for the system (4).

Proof. Define the matrix $|A|_{\epsilon}=\left(\left|a_{j k}\right|+\epsilon\right)$ for $\epsilon>0$. Then $\rho\left(\left|A_{\epsilon}\right|\right)>\rho(|A|)$ and $\rho\left(|A|_{\epsilon}\right) L<1$ for $\epsilon$ small. The matrix $|A|_{\epsilon}$ is positive and it has a positive left eigenvector $\alpha^{\mathrm{T}}=\left(\alpha_{1}, \ldots, \alpha_{n}\right)$. With this vector $\alpha^{\mathrm{T}}$ form the norm (11). In view of

$$
\begin{aligned}
\|f(A y+b)-f(A z+b)\| & \leqslant L\|A(y-z)\| \leqslant L \alpha^{\mathrm{T}}|A(y-z)| \\
& \leqslant L \alpha^{\mathrm{T}}|A|_{\epsilon}|y-z| \leqslant L \rho\left(|A|_{\epsilon}\right) \alpha^{\mathrm{T}}|y-z| \\
& \leqslant L \rho\left(|A|_{\epsilon}\right)\|y-z\| .
\end{aligned}
$$

Thus the mapping $y \mapsto f(A y+b)$ is a global contraction. This shows (i) and (ii). Now consider equation (4). One can assume $\tau=1$. Let $\bar{x}$ be the unique stationary solution and let $x(t)$ be any other solution. Define the measurable functions $d_{j}(t)$ by

$$
d_{j}(t)= \begin{cases}\frac{f_{j}\left(x_{j}(t)\right)-f_{j}\left(\bar{x}_{j}\right)}{x(t)-\bar{x}_{j}} & \text { if } x_{j}(t) \neq \bar{x}_{j} \\ 0 & \text { if } x_{j}(t)=\bar{x}_{j}\end{cases}
$$

and the matrix $D(t)=\left(d_{j}(t) \delta_{j k}\right)$. Then the function $y(t)=(x(t)-\bar{x}) e^{t}$ satisfies the linear equation

$$
\dot{y}=A D(t) y .
$$

A simple argument (see [11]) yields $\|y(t)\| \leqslant$ constant $\times \exp ((\rho(|A|)+\epsilon) L t)$ for any small $\epsilon>0$. 
At any point $x$ where $f^{\prime}(x)$ exists, the Jacobian $J(x)$ of the right-hand side of (3) exists and is given by $A f^{\prime}(x)$ where $f^{\prime}$ is the diagonal matrix $\left(f_{j}^{\prime}\left(x_{j}\right) \delta_{j k}\right)$. Similarly, the Jacobian of $(15)$ is $(1 / \tau)\left(A f^{\prime}(x)-I\right)$.

Proposition 4. Let $\bar{x}$ be a stationary point of the systems (3) and (4) such that $f^{\prime}(\bar{x})$ exists. If the spectral radius of the matrix $A f^{\prime}(\bar{x})$ is less than 1 then the point $\bar{x}$ is a local attractor of the system (3) as well as of the system (4).

Proof. Since the spectrum is in the unit circle, stability in the discrete time case follows. In the continuous time case observe that the spectrum of $A f^{\prime}(\bar{x})-I$ is located in the left half-plane.

Proposition 5. Let $A$ be symmetric and assume that the functions $f_{j}$ are strictly increasing. If $f^{\prime}(\bar{x})$ exists then the eigenvalues of $A f^{\prime}(\bar{x})$ are real.

Proof. $A$ is a symmetric and $f^{\prime}(\bar{x})$ is symmetric and positive definite. Thus the eigenvalues of the product are real.

The contraction property in proposition 3 is just a suffient condition for the existence and uniqueness of the stationary state. In the case of piecewise linear mappings one can obtain stronger results as will be shown in section 6 .

\section{Quasimonotone systems}

We start from a general differential equation $\dot{x}=F(x)$ where $F: \mathbb{R}^{n} \rightarrow \mathbb{R}^{n}$ is continuously differentiable. Let $\mathbb{R}^{n}$ be endowed with the partial ordering induced by the cone $\mathbb{R}_{+}^{n}$. With respect to the nonlinear system it is important to distinguish between several notions of positivity and invariance.

In some situations it is important to know whether non-negative solutions stay nonnegative, i.e. whether the first orthant $\mathbb{R}_{+}^{n}$ in the state space $\mathbb{R}^{n}$ is positively invariant with respect to the flow. Necessary and sufficient condition for this property to hold is that the vector field $F$ is 'inward' on the boundary of $\mathbb{R}_{+}^{n}$, i.e. that $x \geqslant 0, x_{i}=0$ implies $F_{i}(x) \geqslant 0$.

Here we are interested in a quite different notion of positivity which is connected to the positivity of the tangent vector of the flow rather than to the state.

Let $F^{\prime}(x)$ be the derivative (Jacobian) of the function $F$ at the point $x$ (not necessarily a stationary point). $F^{\prime}$ can be represented as the matrix of partial derivatives $F^{\prime}=\left(\partial F_{j} / \partial x_{k}\right)$, depending on $x$. Suppose $F$ has the property that

$$
\frac{\partial F_{j}}{\partial x_{k}}(x) \geqslant 0 \quad \text { for } k \neq j \text { and all } x .
$$

Then the system is called cooperative. Similarly, if

$$
\frac{\partial F_{j}}{\partial x_{k}}(x) \leqslant 0 \quad \text { for } k \neq j \text { and all } x
$$

then the system is called competitive. If the system is either cooperative or competitive then it is called quasimonotone. If the system is competitive then the system $\dot{x}=-F(x)$ is cooperative and vice versa. 
Cooperative systems have several important properties. The most basic is the following. If, for some $t_{0}$, the tangent vector $\dot{x}\left(t_{0}\right) \in \mathbb{R}_{+}^{n}$, then $\dot{x}(t) \in \mathbb{R}_{+}^{n}$ for all $t \geqslant t_{0}$. A first implication is that $x(t)$ is monotone, i.e. each component is non-decreasing. Thus either $x(t)$ does not stay bounded, or it converges to a point. Thus, in a cooperative system with bounded trajectories, $\dot{x}\left(t_{0}\right) \geqslant 0$ implies that the limit set is a point.

Now consider the system (5). The following observation follows from the monotonicity of the function $f$.

Proposition 6. Let the matrix $A$ have the property that $a_{j k} \geqslant 0\left(a_{j k} \leqslant 0\right)$, for $j \neq k$. Then the system (5) is cooperative (competitive).

Probably the most fundamental result on quasimonotone systems is the 'flatness' of limit sets. To understand this result we need several definitions. Two points $x, y \in \mathbb{R}^{n}$ are called related if either $x<y$ or $y<x$.

Theorem $7[15,16]$. Let the system be cooperative or competitive. Let $\omega$ be a limit set. Then $\omega$ does not contain two related points.

From this result follows another important observation.

Theorem $8[15,16]$. Let the system be cooperative or competitive. Let $p>0$ be a positive vector and let $H_{p}=\left\{x: p^{\mathrm{T}} x=0\right\}$ the hyperplane with normal vector $p$. Let $\omega$ be a limit set. Let $P$ be the projection onto $H_{p}$ along $p$. Let $\tilde{\omega}=P \omega$. Then the mapping $P: \omega \rightarrow \tilde{\omega}$ is one-to-one. The set $\tilde{\omega}$ is a limit set of an $(n-1)$-dimensional system.

The theory of quasimonotone systems can be adapted to the discrete time case. Such systems (actually the period maps in periodic differential equations) have been studied by de Mottoni and Schiaffino [26] and by Hale and Somolinos [12]. Let $\mathbb{R}^{n}$ be endowed with the usual coordinates. Consider a mapping $G: \mathbb{R}^{n} \rightarrow \mathbb{R}^{n}$. The corresponding dynamical system $x^{t+1}=G\left(x^{t}\right)$ is called cooperative if the Jacobian $G^{\prime}=\partial G_{j} / \partial x_{k}$ has the property that

$$
\frac{\partial G_{j}}{\partial x_{k}}(x) \geqslant 0 \quad \text { for all } x .
$$

Similarly, if

$$
\frac{\partial G_{j}}{\partial x_{k}}(x) \leqslant 0 \quad \text { for all } x
$$

then the system is called competitive. For a cooperative system we find that $x^{t} \geqslant x^{t-1}$ implies $x^{t+1} \geqslant x^{t}$. This observation has an elementary though interesting consequence.

Proposition 9. Let the system be cooperative or competitive. Suppose the $\omega$-limit set of some point contains two related points. Then the limit set is a periodic orbit.

Proof. First assume that that the system is cooperative. Let $a, b \in \omega\left(x^{0}\right)$ and $a<b$. Then there are $i<j$ such that $x^{i}<x^{j}$. We can assume $i=0$. Then the sequence $x^{k j}$, $k=0,1, \ldots$ is non-decreasing, it converges to some $\bar{x}^{0}$, similarly $x^{l+k j} \rightarrow \bar{x}^{l}, l=1,2, \ldots$. But $\bar{x}^{j}=\bar{x}^{0}$. In the competetive case just consider the system generated by $G \circ G$ which is cooperative.

Thus any limit set that contains infinitely many points does not contain any related points. 


\section{Classical neural networks, examples}

\subsection{The Hartline-Ratliff model}

Originally this system was introduced to model the network in the component eye of the horseshoe crab Limulus [10, 13, 31]. It has been essential in understanding phenomena like lateral inhibition, edge enhancement, contrast enhancement. Usually the equations are written in the form

$$
y^{t+1}=\vartheta\left(c-B y^{t}\right) .
$$

Here $c$ is an input, $B=\left(b_{j k}\right)$ is a non-negative matrix, $b_{j k} \geqslant 0$, and $\vartheta$ is the rectifier function

$$
\vartheta(y)=y_{+}=\max (y, 0)=\frac{1}{2}(y+|y|) .
$$

Thus the Hartline-Ratliff system is given in the form (3); the equivalent form (5) is

$$
x^{t+1}=c-B \vartheta\left(x^{t}\right) .
$$

The continuous version of the Hartline-Ratliff system is

$$
\tau \dot{x}+x=c-B \vartheta(x) .
$$

The system (26) has been studied in [15, 27].

Corollary 10. The system (16) in general and the Hartline-Ratliff system in particular is competitive. Hence limit sets are flat.

In principle it is easy to determine all stationary points of the system (23). Suppose $y$ is a stationary point. Then $y \geqslant 0$ and, after renumbering coordinates, $y=(u, 0)$ with $u>0$. Choose an appropriate block partition of the matrix $B$ and the vector $c$. Then $u$ is a solution to a linear system $(I+\tilde{B}) u=\tilde{c}$. Thus all possible stationary points can be found by inspecting $2^{n}$ systems of linear equations.

\subsection{The Linsker model}

The Linsker system has been proposed to simulate the primary steps of optical perception. It has the form (5) with the function $f=(f, \ldots, f)$ and

$$
f(x)= \begin{cases}1 & \text { if } x>1 \\ x & \text { if }|x| \leqslant 1 \\ -1 & \text { if } x<-1 .\end{cases}
$$

In concrete applications it is written in the form

$$
y_{j}^{t+1}=f\left(y_{j}^{t}+b-\sum_{k=1}^{n}\left[q_{j k}+\theta_{k}\right] y_{k}^{t}\right) .
$$

In this case $A=I-Q-e \theta^{\mathrm{T}}$ where $I$ is the identity matrix, $Q=\left(q_{j k}\right)$, and $e \theta^{\mathrm{T}}$ is the dyadic product (matrix of rank one) of the column vector $e=(1, \ldots, 1)^{\mathrm{T}}$ and the row vector $\theta=\left(\theta_{1}, \ldots, \theta_{n}\right)$. The continuous version of the Linsker system is

$$
\tau \dot{x}+x=\left(I-Q-e \theta^{\mathrm{T}}\right) f(x)+b .
$$

For fixed $Q$ this system is competitive for non-negative $\theta$ and cooperative for large negative $\theta$. After the publication of Linsker's paper [22] much attention has been paid to the analysis of the dynamical properties of (28) [6-8, 23, 24]. 


\subsection{The obstacle problem and quadratic programming}

The obstacle problem is a classical generalization of the Dirichlet problem, see, e.g., [19]. Let $\Omega \in \mathbb{R}^{m}$ be a bounded domain and let $f, \psi: \bar{\Omega} \rightarrow \mathbb{R}, g: \partial \Omega \rightarrow \mathbb{R}$ be given functions. Find $u: \bar{\Omega} \rightarrow \mathbb{R}$ such that $-\Delta u \geqslant f$ in $\Omega, u \geqslant \psi$ in $\Omega,(-\Delta u-f)(u-\psi)=0$ in $\Omega$, $u=g$ on $\partial \Omega$. In the case $m=2$ one can visualize $u$ and $\psi$ as surfaces. At any point of $\Omega$ either $u$ is 'free' and satisfies the differential equation or it 'touches' and equals $\psi$. One can assume $g=0$. Then define the convex set $K=\left\{u \in H_{0}^{1}(\Omega), u \geqslant \psi\right\}$ and minimize the functional $J(u)=\int_{\Omega}[\nabla u \cdot \nabla u-2 f u] \mathrm{d} x$ on $K$. Under suitable smoothness conditions, the two problems are equivalent. The discrete analogue is the following quadratic optimization problem. Given a positively definite symmetric matrix $C=\left(c_{j k}\right)$, and vectors $d=\left(d_{j}\right)$, $\psi=\left(\psi_{j}\right)$, and let

$$
J(x)=\frac{1}{2} \sum_{j, k=1}^{n} c_{j k} x_{j} x_{k}-\sum_{j=1}^{n} d_{j} x_{j} .
$$

Minimize $J(x)$ under the condition $x \geqslant \psi$. Under suitable conditions the solution can be approximated by the following procedure [3]. Choose a vector $x$ and a coordinate $i$. Solve the one-dimensional variational problem to minimize $J\left(x+\alpha e_{i}\right)$ where $e_{i}$ is the $i$ th coordinate vector. The solution is $z=\left(z_{j}\right)$, where $z_{j}=x_{j}$ for $j \neq i$ and $z_{i}=x_{i}+d_{i}-e_{i}^{\mathrm{T}} A x$. Then determine the new vector according to

$$
\tilde{x}= \begin{cases}x_{j} & \text { if } j \neq i \\ z_{i} & \text { if } j=i \text { and } z_{i}>\psi_{i} \\ \psi_{i} & \text { if } j=i \text { and } z_{i} \leqslant \psi_{i} .\end{cases}
$$

In other words

$$
\tilde{x}_{i}=\max \left(\psi_{i}, x_{i}+d_{i}-\sum_{k=1}^{n} c_{i k} x_{k}\right) .
$$

We introduce a new variable $y=x-\psi$. Then the system assumes the form

$$
y^{t+1}=f\left(A y^{t}+b\right)
$$

with $A=I-C, b=d+C \psi, f(y)=y_{+}$. Thus we have shown that the discrete obstacle problem is a network system that is a direct generalization of the Hartline-Ratliff system.

\subsection{Sigmoid dynamics}

It appears that the dynamical system (3) or (5), with a general matrix $A$ (no assumptions an the signs of the entries nor symmetry) and general, though increasing, functions $f_{i}$, has no special properties (see section 6, however).

\section{Uniqueness of the stationary solution}

Under the hypothesis of proposition 1 there is a unique stationary state for every input $b$. In particular the stationary state is unique, i.e. the mapping $H: \mathbb{R}^{n} \rightarrow \mathbb{R}^{n}, H(x)=x-A f(x)$ is univalent. The hypothesis of proposition 1 is close to being optimal with respect to 
contractivity, but not with respect to univalence. The problem of univalence can be approached in a general way. Let $\Omega \subset \mathbb{R}^{n}$ be an open domain. $H: \Omega \rightarrow \mathbb{R}^{n}$ be a continuously differentiable mapping. If the Jacobian $H^{\prime}(x)$ is non-singular at a point $x \in \Omega$ then $H$ is locally invertible (in a neighbourhood of $x$ ). However, if $n>1$, then there are examples where $H^{\prime}(x)$ is invertible for every $x \in \Omega$ and nevertheless the map $H$ is not univalent, even if the set $\Omega$ is convex.

The theorem of Gale and Nikaido gives a sufficient condition for univalence that is well suited to neural networks. The set $\Omega$ called an open rectangle (or an open order interval) if $\Omega=\left\{x=\left(x_{j}\right): p_{j} \leqslant x_{j} \leqslant q_{j}, j=1, \ldots, n\right\}$ where $p, q \in \mathbb{R}^{n}$ with $p_{j}<q_{j}$ for $j=1, \ldots, n$. A matrix is called a $P$-matrix iff all its principal minors are positive. A matrix is called a weak $P$-matrix if its determinant is positive and all its (other) principal minors are non-negative.

Theorem 11 [9]. Let $\Omega$ be an open rectangle, let $H: \Omega \rightarrow \mathbb{R}^{n}$ be continuously differentiable. Assume that $H^{\prime}(x)$ is a weak $P$-matrix for all $x \in \Omega$. Then $H$ is univalent.

We apply this theorem to equation (18).

Proposition 12. Let the function $f$ be continuously differentiable. Assume that for all $x \in \mathbb{R}^{n}$ the matrix $I-A f^{\prime}(x)$ is a $P$-matrix. Then to every $b \in \mathbb{R}^{n}$ there is at most one stationary point.

Sandberg [29] considers a similar problem for the equation $A x+f(x)=b$. Essentially he shows that $x \mapsto A x+f(x)$ is univalent if $A$ is a weak $P$-matrix and $f$ is increasing. This result can be derived from the Gale-Nikaido theorem by observing that the sum of a weak $P$-matrix and a non-negative diagonal matrix is a weak $P$-matrix. For lack of differentiability one cannot directly apply the Gale-Nikaido theorem to piecewise linear mappings. We infer a result of Samelson et al [28] in the form given by Kuhn and Löwen $[11,20]$. Let $S, T$ be any real matrices of order $n$. Define the mapping $g: \mathbb{R}^{n} \rightarrow \mathbb{R}^{n}$ by

$$
g(x)=S x_{+}-T x_{-} .
$$

Proposition 13 [28]. The following assertions are equivalent:

(i) $g$ is injective.

(ii) $g$ is bijective.

(iii) Let $P$ denote any diagonal matrix with diagonal entries from $\{0,1\}$. The determinants

$$
\operatorname{det}(S P+T(I-P)))
$$

are all non-zero and have the same sign.

(iv) $S$ and $T$ are invertible, and all principal minors of $S^{-1} T$ (and of $T^{-1} S$ ) are positive.

This result can be applied to several problems, e.g. the Hartline-Ratliff model. The equation $x=c-B x$ for stationary solutions can be written $(I+B) x_{+}-I x_{-}=c$, hence it assumes the form (29) with $S=I+B, T=I$. Thus we have the following result.

Proposition 14 [11]. The following two assertions are equivalent.

(i) All principal minors of the matrix $I+B$ are positive.

(ii) For any input $c$ the Hartline-Ratliff system has a unique stationary point. If the matrix

$B$ is symmetric then the following statement is equivalent to (i) or (ii)

(iii) The matrix $I+B$ is positively definite. 
The uniqueness problem for the Linsker system is more involved. The equation for stationary points is (18) with $f$ given by (25). Thus one has to study the mapping $g(x) \equiv x-A f(x)$ and solve the equation $g(x)=b$. The hyperplanes $\left\{x: x_{j}=+1\right\}$ and $\left\{x: x_{j}=-1\right\}$ divide $\mathbb{R}^{n}$ into a chamber system $\mathcal{C}$ of $3^{n}$ 'chambers'. This chamber system has branching number $b(\mathcal{C})=4$. The branching number is the smallest number $b$ such that each face of codimension 2 has at most $b$ neighbouring chambers (see [20]). On a given chamber each of the coordinates $x_{j}$ is either less than -1 or between -1 and +1 or greater than +1 . The function $g: \mathbb{R}^{n} \rightarrow \mathbb{R}^{n}$ is a piecewise affine mapping, i.e. $g$ is well-defined and on each chamber $g$ is an affine mapping. Consider a fixed chamber. Assume the coordinates are numbered such that the first group satisfies $x_{j}<-1$, the second group $-1 \leqslant x_{j} \leqslant 1$ and the third group $x_{j}>1$. Define the corresponding block partition of the matrix $A$ and of the vector $x$. Then

$$
\begin{aligned}
& x-A f(x)=\left(\begin{array}{l}
x_{1} \\
x_{2} \\
x_{3}
\end{array}\right)-\left(\begin{array}{lll}
A_{11} & A_{12} & A_{13} \\
A_{21} & A_{22} & A_{23} \\
A_{31} & A_{32} & A_{33}
\end{array}\right)\left(\begin{array}{c}
-e \\
x_{2} \\
e
\end{array}\right) \\
& x-A f(x)=\left(\begin{array}{ccc}
I & -A_{12} & 0 \\
0 & I-A_{22} & 0 \\
0 & -A_{32} & I
\end{array}\right)\left(\begin{array}{l}
x_{1} \\
x_{2} \\
x_{3}
\end{array}\right)-\left(\begin{array}{l}
-A_{11} e+A_{13} e \\
-A_{21} e+A_{23} e \\
-A_{31} e+A_{33} e
\end{array}\right)
\end{aligned}
$$

where $I$ denotes the identity matrix of any order and $e=(1, \ldots, 1)^{\mathrm{T}}$. A mapping $g: \mathbb{R}^{n} \rightarrow \mathbb{R}^{n}$ is called proper if for any sequence $z_{k} \in \mathbb{R}^{n}$ with $\left\|z_{k}\right\| \rightarrow \infty$ also $\left\|g\left(z_{k}\right)\right\| \rightarrow \infty$. In the present case the function $g$ is proper since the term $A f(x)$ is bounded. A piecewise affine mapping is called coherently oriented if on each chamber the determinant of the affine map is non-zero and if all these determinants have the same sign. In the present case one has to check the determinant of the matrix in (33). This determinant is equal to $\operatorname{det}\left(I-A_{22}\right)$ and this is a principal minor of the matrix $I-A$.

We use a result from [20] that extends results of Schramm [30].

Proposition 15 [20]. Let $g$ be a piecewise affine mapping on a chamber system $\mathcal{C}$ with branching number $b(\mathcal{C}) \leqslant 4$. Then the following are equivalent.

(i) $g$ is coherently oriented and proper.

(ii) $g$ is bijective.

From this proposition we infer the following theorem.

Theorem 16. The following two assertions are equivalent:

(i) All principal minors of the matrix $I-A$ are positive.

(ii) For any input $b$ the Linsker system has a unique stationary point. For a symmetric matrix $A$ the following statement is equivalent to (i) or (ii):

(iii) The matrix $I-A$ is positively definite.

If the matrix $A$ has the form $A=I-Q-e \theta^{\mathrm{T}}$ then one has to check the principal minors of the matrix $Q+e \theta^{\mathrm{T}}$. 


\section{Asynchronous dynamics}

In equations (3), (5) we have considered dynamical systems in the classical sense. These dynamical systems have the form of networks: There is a natural choice of coordinates $j=1, \ldots, n$. By definition the dynamics is synchronous. In these discrete time systems one can also consider asynchronous dynamics. An asynchronous dynamics requires two definitions. First one has to define the local action, i.e. the evaluation of a single neuron. Then one has to specify the process which defines the sequence of evaluation sites. The first step is standard. The evaluation at position $i$ is given by

$$
\left(F_{i}(y)\right)_{j}= \begin{cases}y_{j} & \text { if } j \neq i \\ f_{i}\left(\sum_{k=1}^{n} a_{i k} y_{k}+b_{i}\right) & \text { if } j=i .\end{cases}
$$

For the second step one can specify a stochastic process that ensures that all neurons are evaluated again and again. Here we use the simple procedure that the $i$ th neuron is selected with probability $a_{i}>0\left(\sum_{i} a_{i}=1\right)$. A less simple process that makes repeated evaluation of the same neuron less probable, has been suggested by Hopfield [5, 17].

The notion of dissipativeness can be carried over to the case of asynchronous dynamics. A set $M \in \mathbb{R}^{n}$ is called invariant under the asynchronous dynamics if $F_{i}(M) \subset M$ for $i=1, \ldots, n$. The system is called dissipative with respect to the asynchronous dynamics if for every starting point the sequence of iterates reaches the set $M$ in a finite number of steps a.s.

Proposition 17. Suppose any of the hypotheses of proposition 1 is satisfied. Then the system is dissipative with respect to the asynchronous dynamics.

Proof. Apply the proof of proposition 1 to the individual components. The following observation leads to bounds for the stop time (with respect to arriving in $M$ ) In case (i) the iterate is in $M$ after each neuron has been evaluated at least once. In cases (ii) and (iii) the iterates are in $M$ after 'two full sweeps', i.e. after the following has happened: in a first 'sweep' each neuron has been evaluated at least once to get the lower bound (in case (iii)), in a second sweep each neuron is evaluated once to get the upper bound. We need the following lemma.

Lemma 18. Let $f: \mathbb{R} \rightarrow \mathbb{R}$ be a continuous strictly increasing function. Then for $a, z \in \mathbb{R}$ the following inequality holds:

$$
f(z) z-f(a) z \geqslant \int_{f(a)}^{f(z)} f^{-1}(v) \mathrm{d} v .
$$

Equality holds only for $z=a$.

Proof. It suffices to prove the lemma for continuously differentiable $f$. The general case follows by approximation. The function $F(z)=\int_{z_{0}}^{z} f(u) \mathrm{d} u$ is convex, thus by Taylor's formula

$$
F(z) \geqslant F(a)+F^{\prime}(a)(z-a)
$$




$$
\begin{aligned}
F^{\prime}(a)(z-a) & \leqslant F(z)-F(a) \\
f(a)(z-a) & \leqslant \int_{a}^{z} f(u) \mathrm{d} u \\
& =f(z) z-f(a) a-\int_{a}^{z} f^{\prime}(u) u \mathrm{~d} u
\end{aligned}
$$

by partial integration. Substitute $v=f(u), \mathrm{d} v=f^{\prime}(u) \mathrm{d} u$ to obtain

$$
f(a) z \leqslant f(z) z-\int_{f(a)}^{f(z)} f^{-1}(v) \mathrm{d} v
$$

which is equation (35). The assertion on equality follows by considering the remainder term in Taylor's formula.

Next we consider the special case that $A$ is a symmetric matrix. Define an energy function

$$
V(y)=\sum_{j=1}^{n} \int_{0}^{y_{j}} f^{-1}(s) \mathrm{d} s-\frac{1}{2} \sum_{j, k=1}^{n} a_{j k} y_{j} y_{k}-\sum_{j=1}^{n} b_{j} y_{j} .
$$

Theorem 19. Let $A=\left(a_{j k}\right)$ be symmetric and $a_{j j} \geqslant 0$ for $j=1, \ldots, n$. Then, for $i=1, \ldots, n$,

$$
V\left(F_{i}(y)\right) \leqslant V(y) \quad \text { for } y \in \mathbb{R}^{n} \quad \text { with } y \in f(\mathbb{R}) .
$$

The equality $V\left(F_{i}(y)\right)=V(y)$ holds if and only if $F_{i}(y)=y$.

Proof. Denote $y=\left(y_{j}\right)$ and $F_{i}(y)=\tilde{y}=\left(\tilde{y}_{j}\right)$. Then $\tilde{y}_{j}=y_{j}$ for $j \neq i$ and

$$
\tilde{y}_{i}=f_{i}\left(\sum_{k=1}^{n} a_{i k} y_{k}+b_{i}\right) \text {. }
$$

Using symmetry, the functional $V(y)$ can be written

$$
\begin{gathered}
V(y)=\sum_{j \neq i} \int_{0}^{y_{j}} f_{j}^{-1}(s) \mathrm{d} s-\frac{1}{2} \sum_{j, k \neq i} a_{j k} y_{j} y_{k}-\sum_{j \neq i} b_{j} y_{j}+\int_{0}^{y_{i}} f_{i}^{-1}(v) \mathrm{d} v \\
-\sum_{k \neq i} a_{i k} y_{i} y_{k}-b_{i} y_{i}-\frac{1}{2} a_{i i} y_{i}^{2} \\
V(\tilde{y})=\sum_{j \neq i} \int_{0}^{y_{j}} f_{j}^{-1}(s) \mathrm{d} s-\frac{1}{2} \sum_{j, k \neq i} a_{j k} y_{j} y_{k}-\sum_{j \neq i} b_{j} y_{j}+\int_{0}^{\tilde{y}_{i}} f_{i}^{-1}(v) \mathrm{d} v \\
-\sum_{k \neq i} a_{i k} \tilde{y}_{i} y_{k}-b_{i} \tilde{y}_{i}-\frac{1}{2} a_{i i} \tilde{y}_{i}^{2} .
\end{gathered}
$$

Thus, with $z=\sum_{k} a_{i k} y_{k}+b_{i}, \tilde{y}=f(z)$,

$$
\begin{gathered}
V(\tilde{y})-V(y)=\int_{y_{i}}^{\tilde{y}_{i}} f_{i}^{-1}(v) \mathrm{d} v-\sum_{k \neq i} a_{i k}\left(\tilde{y}_{i}-y_{i}\right) y_{k}-\frac{1}{2} a_{i i}\left(\tilde{y}_{i}^{2}-y_{i}^{2}\right)-b_{i}\left(\tilde{y}_{i}-y_{i}\right) \\
=-\left(f_{i}(z)-y_{i}\right) z+\int_{y_{i}}^{f_{i}(z)} f_{i}^{-1}(v) \mathrm{d} v-\frac{1}{2} a_{i i}\left(\tilde{y}_{i}-y_{i}\right)^{2}
\end{gathered}
$$


There is $a \in \mathbb{R}$ with $y_{i}=f_{i}(a)$. From lemma 18 it follows that $V\left(F_{i}(y)\right) \leqslant V(y)$. Suppose the right-hand side in (40) vanishes. Then the first term vanishes. In view of Lemma $18 z=a$ and $\tilde{y}_{i}=f_{i}(z)=f_{i}(a)=y_{i}, F_{i}(y)=y$. By linear superposition we get the corresponding result for the stochastic selection procedure. Let $y^{t+1}$ be the random variable that is obtained by evaluating the $i$ th unit with probability $a_{i}$. Then

$$
E\left(V\left(y^{t+1}\right) \mid y^{t}\right) \leqslant V\left(y^{t}\right) \quad \text { for } y \in f\left(\mathbb{R}^{n}\right) .
$$

Therefore, the function $V$ is a supermartingale on the set $\left\{y \in \mathbb{R}^{n}: y \in f(\mathbb{R})\right\}$.

A sufficient condition for convergence of $V\left(y^{t}\right)$ a.s. is uniform integrability of the supermartingale. A sufficient condition for the latter property is the existence of a lower bound. In general the function $V$ will not be bounded below. A lower bound can be ensured in several ways. Either one can prove that the system is dissipative (cf proposition 17) and thus restrict the problem to a compact set. Or one can require that the leading term of $V$ grows to $+\infty$ at least quadratic for $\|y\| \rightarrow \infty$. Thus we get the following results. For the system (5) let $S$ be the set of stationary points. For $\epsilon>0$ let $S_{\epsilon}=\cup_{y \in S} B(y, \epsilon)$ where $B(y, \epsilon)$ is the open ball with center $y$ and radius $\epsilon$. For any realization $\left\{y^{t}\right\}$ define the stopping time as $\inf \left\{t: y^{t} \in S_{\epsilon}\right\}$.

Theorem 20. Let $A=\left(a_{j k}\right)$ be symmetric and $a_{j j} \geqslant 0$ for $j=1, \ldots, n$. Let the system be dissipative. Then the stopping time is finite a.s.

Proof. As long as $y^{t} \notin S_{\epsilon}$

$$
g\left(y^{t}\right) \equiv E\left(V\left(y^{t+1} \mid y^{t}\right)\right)-V\left(y^{t}\right)<0 .
$$

Let $C$ be the compact set in the definition of dissipativeness. There is a $\delta=\delta(\epsilon)>0$ such that $-g\left(y^{t}\right)>\delta$ for $y^{t} \in C \backslash S_{\epsilon}$. Hence

$$
M_{t}=V\left(y^{t}\right)+t \delta
$$

is also a lower bounded supermartingale for $y^{t} \in C \backslash S_{\epsilon}$. According to Doob's theorem [4] $M_{\tau \wedge t}$ is a lower bounded supermartingale where $\tau \wedge t=\min \{\tau, t\}$. From the convergence theorem for supermartingales it follows that

$$
\lim _{t \rightarrow \infty} M_{\tau \wedge t}=M<\infty \quad \text { a.s. }
$$

thus

$$
\lim _{t \rightarrow \infty} M_{\tau \wedge t}=\lim _{t \rightarrow \infty}\left(V\left(y^{\tau \wedge t}\right)+\delta(\tau \wedge t)\right)<\infty \quad \text { a.s. }
$$

Note that $V\left(y^{\tau \wedge t}\right)$ is itself a bounded supermartingale, therefore $\lim _{t \rightarrow \infty}(\tau \wedge t)<\infty$ a.s. which implies $\tau<\infty$ a.s.

Corollary 21. (i) Let the matrix in the Linsker system be symmetric, i.e. $Q+\theta e^{\mathrm{T}}$ is symmetric, and $1-q_{j j}-\theta_{j} \geqslant 0$ for $j=1, \ldots, n$. Then the stopping time is almost certainly finite.

(ii) Let the matrix $B=\left(b_{j k}\right)$ of the Hartline-Ratliff system be symmetric (and of course non-negative). Let $b_{j j}=0$ for $j=1, \ldots, n$. Then the stopping time is finite a.s. 
The linear case $f(y)=y$ shows that the symmetry of the matrix $A$ is a sufficient, but not a necessary condition. If the matrix $A$ has non-negative entries and if the selection procedure is cyclic then a necessary and sufficient condition for convergence to equilibrium is $\rho(A)<1$ (the Stein-Rosenberg theorem, see [18]).

In the synchronous dynamics the existence and uniqueness of a stationary point, even if it is a local attractor, is not sufficient to ensure global stability. The behaviour is different with asynchronous dynamics.

Theorem 22. Assume the hypothesis of theorem 20. Further assume that there is exactly one stationary point $\bar{x}$. Then the asynchronous dynamics converges to $\bar{x}$ a.s., i.e. the probability distribution $\mu_{t}$ of $y^{t}$ converges to $\delta_{\bar{x}}$.

Proof. see [21]. The uniqeness of the stationary point has been considered in propositions and theorems 3, 11, 14 .

\section{Acknowledgment}

This paper was partially supported by a grant from the A von Humboldt foundation of Germany, given to JF.

\section{References}

[1] Amit D 1989 Modeling Brain Function: The World of Attractor Neural Networks (Cambridge: Cambridge University Press)

[2] Berman A and Plemmons R J 1979 Nonnegative Matrices in the Natural Sciences (New York: Academic)

[3] Céa J 1971 Optimisation, Théorie et Algorithmes (Paris: Dunod) p 118 ff; 1978 Lectures on Optimization, Theory and Algorithms (Tata Institute of Fundamental Research, Bombay) (Berlin: Springer)

[4] Doob J L 1953 Stochastic Processes (New York: Wiley)

[5] Feng J 1994 The convergence of the Hopfield type model Adv. Math. 23 (4) 451-63

[6] Feng J and Pan H 1993 Analysis of Linsker type Hebbian learning: rigorous results Proc. 1993 IEEE Conf. on Neural Networks vol III pp 1516-21

[7] Feng J, Pan H and Roychowdhury V P 1995 A Rigorous analysis of Linsker's Hebbian learning network Advances in the Neural Information Processing System 7 ed G Tesauro, D Touretzky and T K Leen (Cambridge, MA: MIT Press) pp 319-26

[8] Feng J, Pan H and Roychowdhury V P 1996 On neurodynamics with limiter function and Linsker's developmental model Neural Comput. 8 1003-19

[9] Gale D and Nikaido H 1965 The Jacobian matrix and global univalence of mappings Math. Ann. 159 81-93

[10] Hadeler K P 1974 On the theory of lateral inhibition Kybernetik (Biol. Cybern.) 14 161-5

[11] Hadeler K P and Kuhn D 1987 Stationary states of the Hartline-Ratliff model Biol. Cybern. 56 411-17

[12] Hale J K and Somolinos A J 1983 Competition for fluctuating nutrient Math. Biol. 18 255-80

[13] Hartline H K and Ratliff F 1958 Spatial summation of of inhibitory influence in the eye of Limulus and the mutual interaction of receptor units J. Gen. Physiol. 41 1049-66

[14] Hertz J, Krogh A and Palmer R G 1991 Introduction to the Theory of Neural Computation (Reading, MA: Addison-Wesley)

[15] Hirsch M W 1982 Systems of differential equations which are competive or cooperative I. Limit sets SIAM J. Math. Anal. 13 167-79

[16] Hirsch M W 1985 Systems of differential equations which are competive or cooperative II, Convergence everywhere SIAM J. Math. Anal. 16 423-39

[17] Hopfield J J 1982 Neural networks and physical systems with emergent collective computational abilities Proc. Natl Acad. Sci. USA $792554-8$

[18] Householder A S 1964 The Theory of Matrices in Numerical Analysis (New York: Blaisdell)

[19] Kinderlehrer D and Stampacchia G 1980 An Introduction to Variational Inequalities and their Applications (New York: Academic) 
[20] Kuhn D and Löwen R 1987 Piecewise affine bijections of $\mathbb{R}^{n}$, and the equation $S x_{+}-T x_{-}=y$. Linear Alg. Appl. 96 109-129

[21] Liggett T M 1985 Interacting Particle Systems (Berlin: Springer)

[22] Linsker R 1986 From basic network principle to neural architecture I-III Proc. Natl Acad. Sci. USA 83 7508-12, 8390-4, 8779-83

[23] Linsker R 1986 Self-organization in a perceptual network Computer 21 105-17

[24] Mackay D J C and Miller K D 1990 Analysis of Linsker's application of Hebbian rules to linear networks Network 1 257-97

[25] McCulloch W S and Pitts W 1943 A logical calculus of the ideas immanent in neural nets Bull. Math. Biophys. 5 115-37

[26] de Mottoni P and Schiaffino A 1981 Compettition systems with periodic coefficients A geometric approach. J. Math. Biol. 11 319-35

[27] Morishita I and Yajima A 1972 Analysis and simulation of networks of mutually inhibiting neurons Kybernetik (Biol. Cybern.) 11 154-65

[28] Samelson H, Thrall R M and Wesler O 1958 A partition theorem for Euclidean $n$-space Proc. Am. Math. Soc. 9 805-07

[29] Sandberg I W 1971 Necessary and sufficient conditions for the global invertibility of certain nonlinear operators that arise in the analysis of networks IEEE Trans. Circuit Theory CT-18 260-63 (reprinted Wilson A N Jr (ed) 1975 Nonlinear Networks (Piscataway, NJ: IEEE Press))

[30] Schramm R 1980 On piecewise linear functions and piecewise linear equations Math. Oper. Res. 5 510-22

[31] Varjú D 1962 Vergleich zweier Modelle für die laterale Inhibition Kybernetik (Biol. Cybern.) 1 200-8 